\title{
AR Model Based Human Identification using Ear Biometrics
}

\author{
Farida Khursheed ${ }^{1}$ and A.H. Mir ${ }^{2}$ \\ ${ }^{1,2}$ Department of Electronics \& Communication Engineering \\ National Institute of Technology, Srinagar - 190006 INDIA \\ 1'fklone@nitsri.net, ${ }^{2}$ ahmir@nitsri.net
}

\begin{abstract}
In this paper usefulness of time series based Auto Regressive (AR) modelling technique has been explored for identification of a person. For this purpose, time series is obtained from the contour coordinates of the ear. AR model is fitted to this time series. AR coefficients thus obtained serve as a feature vector. Recognition Rate (RR) has been found by a classifier that is based on Euclidian distance between feature vector of test samples with training samples within itself (intraclass) and with respect to others (interclass). Model has been found invariant to posture, rotation and illumination. RR up to $99 \%$ has been obtained. Results have been compared with existing techniques. The results demonstrate the effectiveness of technique for human identification.
\end{abstract}

Key Words: Ear biometrics, Human identification, Time series modelling, AR modelling, Recognition

\section{Introduction}

Human identification has been a subject of intensive research for the few decades because of its applications in almost all aspects of secure surveillance.

In today's world when threats to individual, critical infrastructure, personal data and financial data have become imminent, identity of a person has become of a paramount importance. Increased battle of cyber security threats with conventional security methods of personnel identification has given rise to biometric security systems. As certain human traits can not be stolen or modified, use of biometrics has been a subject of great importance for personal identification.

The biometric security system establishes human identity using an individual's physical or behavioural attributes such as face, iris, voice or gait.

A biometric system is a recognition system which operates by acquiring biometric data from an individual. The biometric data extracted has to be invariant and computationally stable. Feature vector is extracted from the prescribed data sets. The feature vectors thus obtained are compared with template sets in the data base to establish the match. Most of the current research in biometrics for verification and identification is found on, iris, fingerprint, face, hand geometry, gait; in view of the availability of their invariant biometric feature vectors. Surveys on these technologies are available in [1-5].

It may be pointed out that even if any physiological or behavioural biometric characteristics are universal, unique, permanent, it may not be usable because of its unacceptability. Therefore while selecting a biometric feature it is necessary to give due consideration to acceptability. In this sense, ear is one of the most suitable candidate to be used for biometrics. In addition, it does not change with emotions, states of mind, sadness, fear or cosmetic changes. Lastly ear can be easily captured from a distance, even if the subject is not fully cooperative. This makes ear recognition especially interesting for smart surveillance tasks and for forensic image analysis as well. Besides, it may be realized that, most of the biometrics systems nowadays do not live up to their expectations, usually due to the requirements of well controlled environments. This is one 
of the reasons that motivated us to use time series modelling that is only dependent on structural information depicted by outer boundary of the ear that is unlikely to change with change in illumination.

\subsection{Related Work}

Ear biometric has gained attention for more than ten years [6]. During crime scene investigations, ear marks are often used for identification in absence of valid fingerprints. The advantages of using ear as a source of data for human identification compared to other parts of the body are given in [7, 8]. Presently, police and forensic specialists use ear prints as a standard proof of identification [9-13].

One of the first ear biometric systems was introduced by Iannaralli. In this system 10,000 ears were examined and distance between predicted points of human ear was measured. However the limitation of this approach is that it requires accurate estimation of a stable reference points for measurement which is very difficult in real environment. Since then, a number of 2D automatic ear recognition methods have been proposed [14], [15]. 2D ear recognition approaches have been divided into four different subclasses.

\subsubsection{Holistic Approaches}

In Holistic approach [16], [17] used force field transformation for ear recognition. Pose invariance has been added to edges extracted by using the force field method as given in [18]. Kumar A. and $\mathrm{Wu}$. [19] presented an ear recognition approach which uses the phase information of log-Gabor filters for encoding the local structure of the ear. Abate et al., [20] used a generic Fourier descriptor for rotation and scale invariant feature. Wang et al., [21] composed six different feature vectors by using seven moment invariants.

\subsubsection{Local Approach}

In Local approach an automated geometrical approach has been proposed in [22, 23]. In this approach ear contour and centroid is extracted from ear image and then constructed concentric circles using that centroid. Scale invariant feature transform(SIFT) is known to be a robust way for landmark extraction even in images with small pose variations and varying brightness conditions as given in $[24,25]$. Bustard et al., showed that SIFT can handle pose variations up to $20^{\circ}$. Extraction of Local Binary patterns (LBP) is a technique for feature extraction on the pixel level. Guo et al., [26] extracted LBP form the raw images and created histograms describing the distribution of the local LBP. Wang and Yan [27] reduced the dimensionality of the feature vector with linear discriminate analysis before a Euclidean distance measure was used to quantify the similarity of two feature vectors.

\subsubsection{Hybrid Approach}

In Hybrid approach Liu et al., [28] Combined front and backside view of the ear by extracting features using the triangle ratio method and Tchebichef moment descriptors. Yuan L and Mu Z, [29]; Youdong Z, and Yunde J, [30] used the active shape model for extracting the outline of the ear.

\subsubsection{Statistical Approach}

In statistical approach Zhang $\mathrm{H}$ and $\mathrm{Mu} \mathrm{Z}$, [31] conducted studies on the effectiveness of statistical methods in combination with classifiers. Xie and MU [32] proposed an improved locally linear embedding (LLE) algorithm for reducing the dimensionality of ear features. A general classification algorithm based on the theory of compressive 
sensing has been used in [33] A complete survey of all the approaches of ear biometrics is given in $[34,35]$.

Literature survey reveals that despite intensive research work in the field of ear biometrics no adequate attempt has been made to explore potential of model based approach for ear recognition. In particular, no attempt has been made to explore the potential of time series modelling for biometric recognition.

\subsection{Our Work}

In this paper, an attempt has been made to check the efficacy of time series modelling technique namely autoregressive modelling for recognition of humans on the basis of ear biometrics. Ear shape is used as a foundation for application of AR model. Time series for application of AR model is obtained from closed contour coordinates of the ear. To obtain these coordinates, ear substructure is isolated from the given image using cropping. Canny edge detector is applied to obtain edges that include the edges of outer boundary. Edge detection is followed by binarization. This is important to make the image suitable for boundary tracing. The coordinates of closed boundary form the time series to which AR model is fitted. The model coefficients thus obtained are used as feature vector that represent the structural information of the ear.

Keeping order of model as variable, these AR coefficients are computed from ear in three different postures and with different rotations with respect to reference. For any subject of interest, feature vector consisting of AR coefficients corresponding to any order can be divided into two sets namely training set and testing set. Corresponding to every subject training and testing sets are compared on the basis Euclidean distance classifier to determine the match.

\section{Edge Detection}

There are number of edge extraction techniques based on gradient measures. The fundamental limitation in these techniques is that the gradient operators are basically point operators and thus do not take into account noise characteristics which are statistical in nature. Moreover, the gradient operation is itself noise sensitive. Some statistical approaches have been proposed for edge detection in radiographic images [36], but the algorithm used there is based on bimodality test of pixels within each window. It has been reported by Haralick RM, [37] that the algorithm works poorly with moderate noise producing a highly broken up segmentation. Directional operators by Navita and Babu Navatia R and Babu KR [38] and Binford [39] introduce implicit averaging which is more along the edge rather than across it. Isotropic operators suggested by Marr and Hilderth [40] and Shanmugam KS, Dicky FM, and Green JA, [41] offer simplicity at the expense of smoothing across the edge. Therefore, Canny's technique [42] has been used for edge detection purpose. Two common criteria relevant to this edge detection techniques are: (i) low error rate, i.e., the edges that occur in images are not missed and there is no spurious response and (ii) localization of edge points, i.e., the distance between the points marked by edge detector and the center of the true edge, is minimized. In addition to these, another criterion has been added to ensure that there is only single response for an edge. Since the optimal response is critically dependent on accuracy of the noise estimate, it has been formulated as an optimization problem. For computational simplicity this optimal operator is replaced by the first order derivative of the Gaussian function. The operator width $(\sigma)$ of the Gaussian function can be varied to locate edge points of different strength.

\subsection{Edge-Detection Technique}

Let $\mathrm{G}$ be a 2-D Gaussian function and $G_{n}$ be the first order derivative of $\mathrm{G}$ in some direction, $\mathrm{n}$. Then $\mathrm{G}$ and $G_{n}$ are given by 


$$
\begin{array}{r}
\mathrm{G}=\exp \left(-\frac{x^{2}+y^{2}}{2 \sigma}\right) \\
G_{n}=\frac{\partial G}{\partial n}=n \cdot \Delta G
\end{array}
$$

Where $x$ and $y$ stand for pixel coordinates and $\mathrm{n}$ can be estimated from the smoothed gradient direction given by:

$$
\mathrm{n}=\frac{\text { WG:I }}{\| \nabla \mathrm{G}: \mathrm{I} \mid}
$$

An edge point is defined to be a local maximum of the operator $G_{n}$ applied to the image I. At the local maximum we have

$$
\frac{\partial}{\partial n}\left(G_{n} * I\right)=0
$$

Substituting $G_{n}$ from Eq. (1), Eq.(3) becomes:

$$
\frac{\partial^{\mathrm{x}}}{\partial n^{\mathrm{x}}}(G * I)=0
$$

At such an edge point, the edge strength will have the magnitude:

$$
\left\|G_{n} * I \rrbracket=\right\| \nabla(G \approx I) \rrbracket
$$

Because of the associativity property of convolution, we convolve the image I, with a Gaussian function, $\mathrm{G}$ and then compute the directional second order derivative zeros so as to locate edges as given by Equation(4) and use the magnitude of Eq.(5) to estimate the edge strength. Depending on the value of $\sigma$, different edge points are obtained.

\section{Time Series Modelling}

An observed record of stochastic process, when ordered chronologically with respect to some index variable is called a time series. The contour can be considered as a series of large number of straight line segments, say $\mathrm{N}$. If $\mathrm{N}$ is large the desired accuracy of the approximation can be obtained. Therefore, the sequence of $\mathrm{N}$ boundary points $\left(x_{i} y_{i}\right),\{$ $=1,2, \ldots \ldots . . . \mathrm{N}-1\}$ constitutes time series. Points on bounding curve of any substructure can be considered as a time series. The parameters of AR model which represent 2D shapes of the substructures can be obtained from the contour points.

Originally developed as useful tool to describe and analyze 1-D discrete time signals as in Poulos M.et al.,[43], Paranjape R. B. et al.,[44]. 2-D applications of the AR model were first proposed by Kashyap and Chellpa [45] who used the model for shape storage, transmission and reconstruction. Dubois and Glanz [46] investigated the usefulness of this model for representing shapes of different pattern sets. Mir et al., [47] used AR model for shape description of human organs in medical images. In this paper we propose to use time series based AR model for identification of a person. The time series for this purpose is obtained from ear contour that represent structural information of the ear shape.

\subsection{Autoregressive Model}

In this approach, the time series constitutes an ordered sets of length of $\mathrm{N}$ boundary points from the centroid.

Let $y_{j}$ be the length of a vector between the boundary points and centroid. The real AR model is formed from the sequence of $y_{j}$ 's as: 


$$
y_{j}=\sum_{k=1}^{m} a_{k} y_{j-k}+w_{j}
$$

This model is based on parameters $a_{k}$ where $\mathrm{k}=1, \ldots, \mathrm{m}$, and $\mathrm{m}$ is the order of the model and $\mathrm{w}_{\mathrm{j}}$ is the error term.

The AR coefficients can be estimated in many ways such as ordinary least square procedure, Markove chain Monte-Carlo method and methods of moments (through YuleWallker equation). There is a direct correspondence between these parameters and the covariance function of the process and this correspondence can be inverted to determine the parameters from the autocorrelation function. This is done by Yule- walker AR method, also called the autocorrelation method to fit the AR model.

Multiplying (1) by $y_{j-1}, y_{j-2}$ and so on and taking the Expectation, the following Yule-Walker equations are obtained:

$$
r_{\mathrm{l}}=\sum_{k=1} a_{k} \widetilde{T}_{\mathrm{l}}-k
$$

Where $\mathbb{l}=0 \ldots \ldots . ., \mathrm{m}$ yielding $\mathrm{m}+1$ equations. Hence $r_{\mathbb{R}}$ is the autocovariance function of $y_{j}$,

Using the evenness of the autocovariance $r_{R}=r_{-l}=E\left\{y_{j} y_{j-1}\right\}$

Rewriting equation (2)

$$
\begin{gathered}
r_{1}=a_{1} r_{0}+a_{2} T_{1}+a_{3} r_{2}+\ldots+a_{m-1} r_{m-2}+a_{m} r_{m-1} \\
r_{2}=a_{1} r_{1}+a_{2} r_{0}+a_{3} r_{1}+\ldots+a_{m-1} r_{m-3}+a_{m} r_{m-2} \\
\vdots \\
\vdots \\
r_{m-1=} a_{1} r_{m-2}+a_{2} r_{m-3}+a_{3} r_{m-4}+\cdots+a_{m-1} r_{0}+a_{m} r_{1} \\
r_{m=}=a_{1} r_{m-1}+a_{2} r_{m-2}+a_{3} r_{m-3}+\cdots+a_{m-1} T_{1}+a_{m} r_{0}
\end{gathered}
$$

This can be also written as

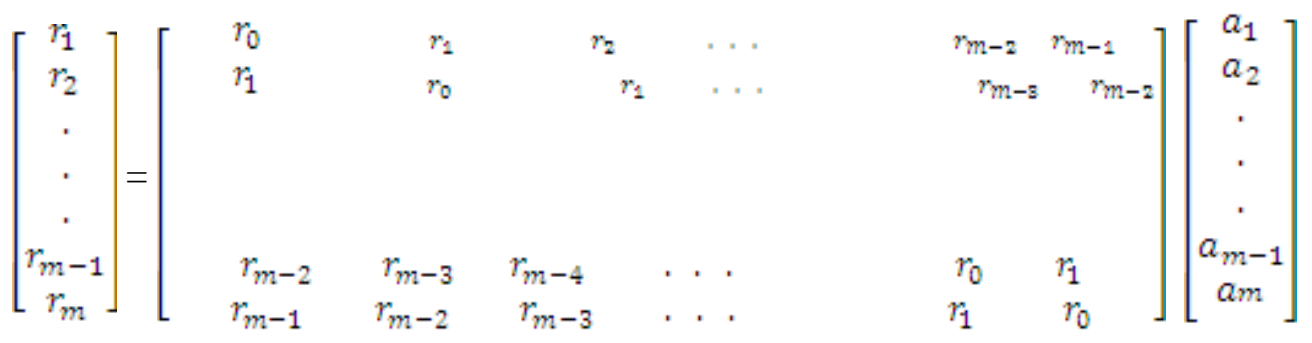

For $r_{0}=1$; 


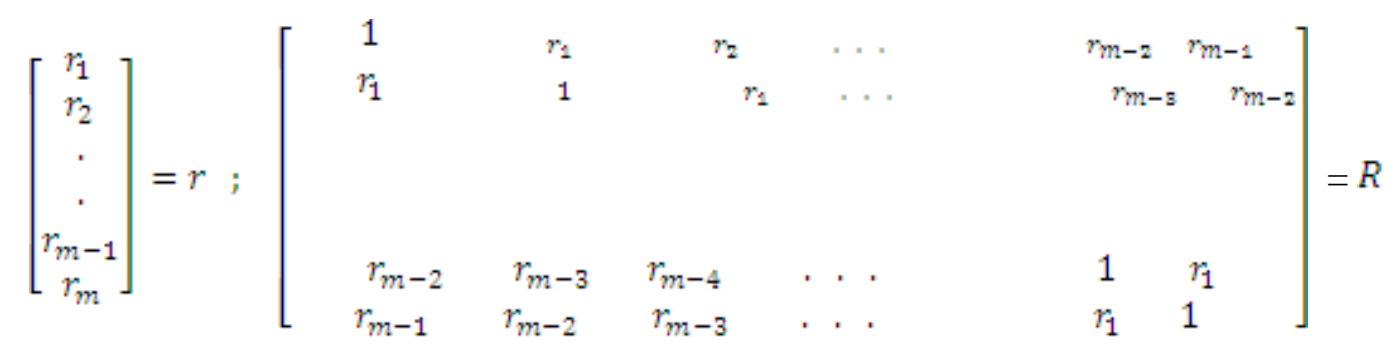

$$
\left[\begin{array}{c}
a_{1} \\
a_{2} \\
\cdot \\
\cdot \\
a_{m-1} \\
a_{m}
\end{array}\right]=a
$$

Therefore we can write as

$$
r=R a
$$

Where $R$ is full-rank and symmetric so that invertability is guaranteed.

Or

$$
a=R_{A R}^{-1}(\mathrm{~m}) r
$$

The coefficients $\left\{a_{k}\right\}$ which form the feature vector are obtained from equation (5).

\section{Implementation}

\subsection{Test Data}

Test ear images have been downloaded from IIT Delhi, India ear database. The data base contains 363 images taken from 121 subjects in three different postures. The subjects in the database are in the age group 14-58 years. The resolution of image database is 272 X 204 pixels and all these images are available in JPEG format. Typical images are shown in FIGURE 1. Processing of these images has been done using MATLAB version 7.9 for implementation of the proposed methodology.

Implementation involves computation of AR coefficients which can be achieved in two steps: a) Extraction of boundary points that are used as an input to AR model and b) Determination of AR coefficients.

\subsubsection{Extraction of Boundary Points}

Extraction of boundary points is done in following steps:

i) Cropping ii) Edge detection iii) Binarization iv) Boundary Tracing

\subsubsection{Cropping}

Test images given in the database are cropped to get isolated ear substructure as shown in Figure $2 \mathrm{~B}$. 


\subsubsection{Edge Detection}

Canny edge detector is used for edge detection of the cropped image as shown in FIGURE 2C. Canny's edge detector detects edges that apart from real edges contain noisy edges. These edges need to be removed leaving only the edge of interest from which boundary can be traced which gives the structural information of the ear image.
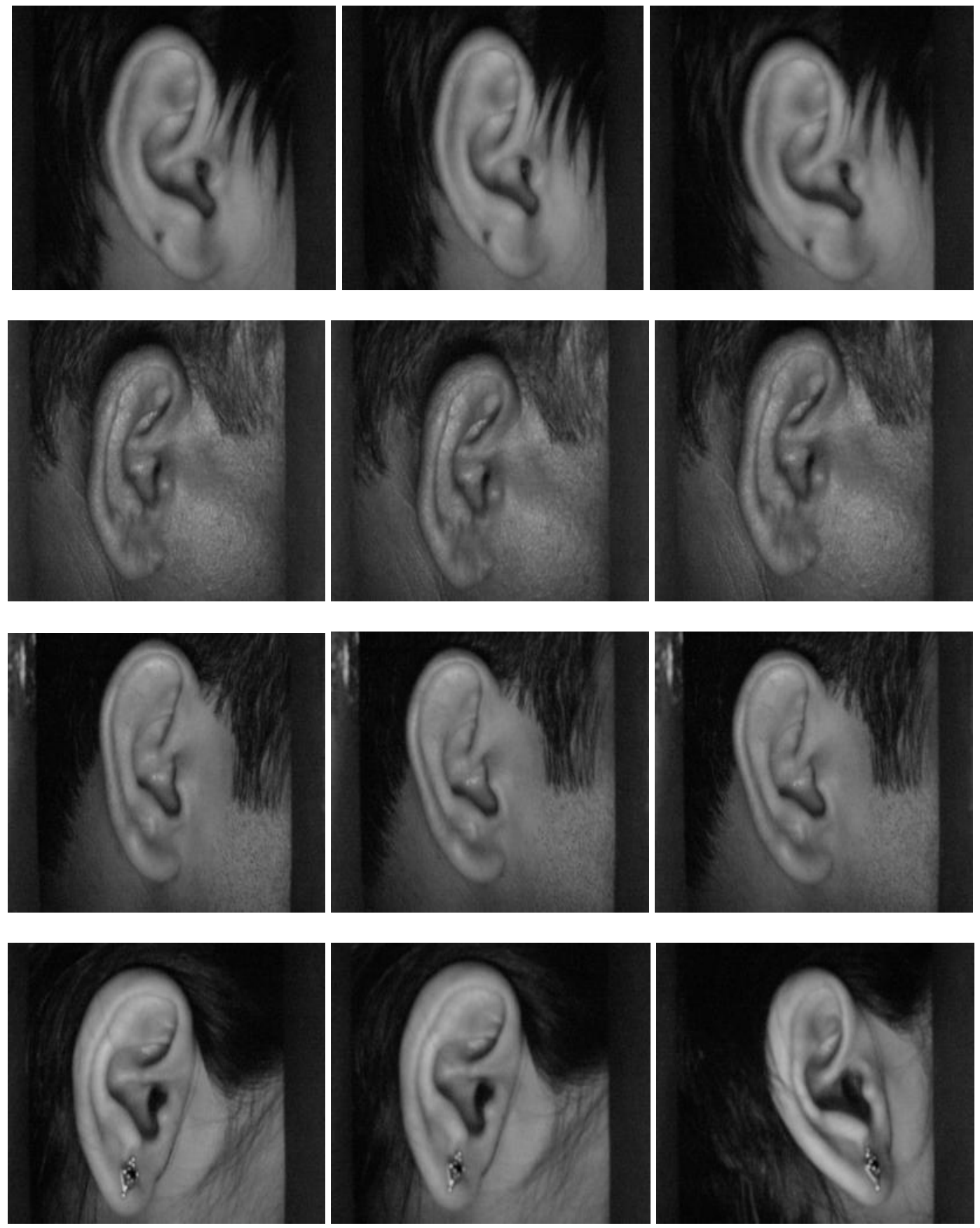

Figure 1. Sample Images of Five Subjects from our Acquired Database 


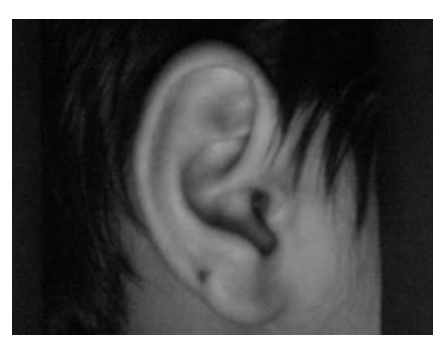

A)

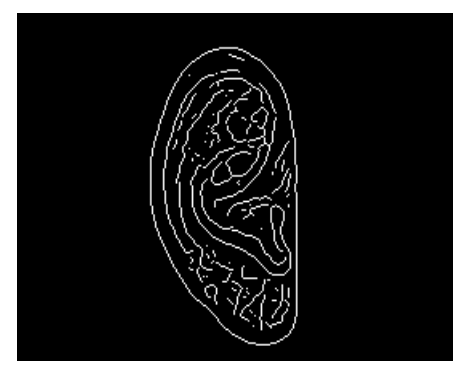

C)

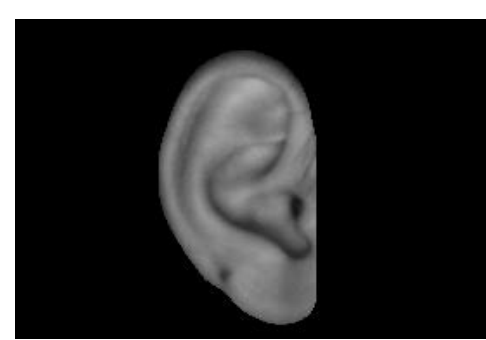

B)

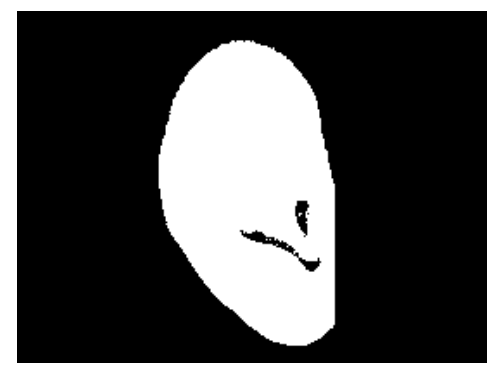

D)

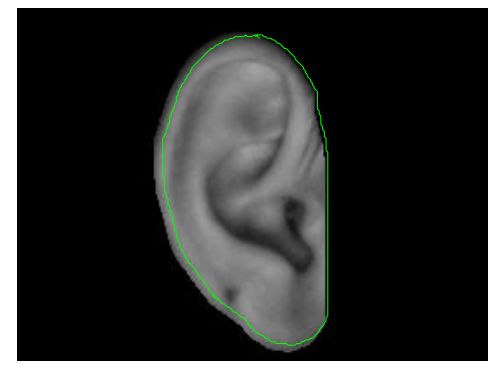

E) $0^{0}$

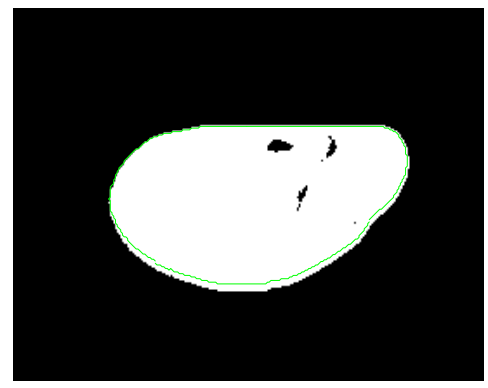

F) $90^{\circ}$

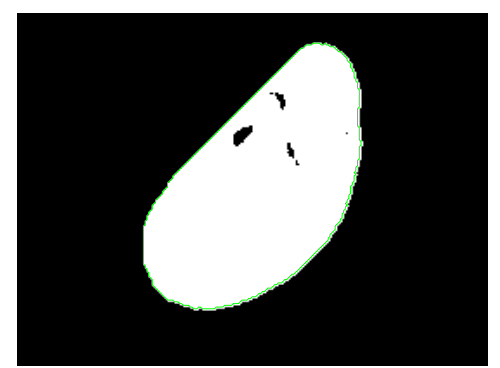

H) $45^{\circ}$

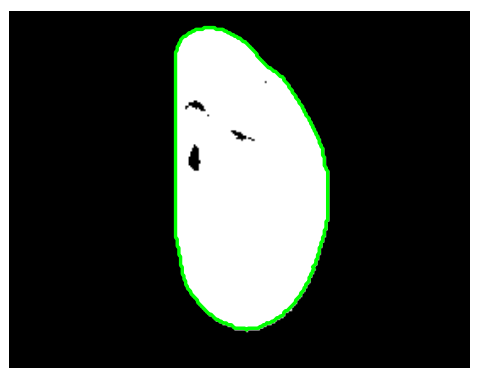

G) $180^{\circ}$

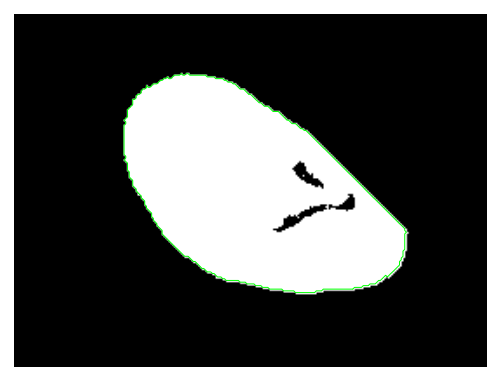

I) $135^{\circ}$

Figure 2. (A) Acquired Image (B) Cropped Image (C) Edge Detected Image (D) Binary Image

E, F, G, H, I ) Contours of: reference image, at rotations : $0^{0}, 90^{\circ}, 180^{\circ}, 45^{\circ}, 135^{\circ}$ 


\subsubsection{Binarization}

The step edge image thus obtained is thresholded to get a binary image as shown in FIGURE 2D. In the methodology, threshold selection is automatic. Threshold is selected on the basis of minimum grey level of the given image. Accordingly all pixels above minimum become white and all other pixels become black. This gives rise to binary picture corresponding to an ear image under consideration. Binarization is important because this makes contour tracing of outer ear boundary simple by creating a transition in grey level for initiation of boundary tracing. Thus binary image is used as an input for boundary tracing.

\subsubsection{Boundary Tracing}

It may be noted that the time series of a finite number $\mathrm{N}$ of distinct observations of $(\mathrm{N}+\mathrm{t})$ th observations , $\mathrm{Y}_{\mathrm{n}+\mathrm{t}}$ will be equal to $\mathrm{Y}_{\mathrm{t}}$. Consequently the time series is cyclic in nature and cycle can be repeated any number of times. An important implication of this fact is that when AR model is fitted to such a series it will be noncausal. In other words concept of past \& future observations becomes irrelevant here and the model fitted to such observations is independent of any starting point or reference point of the contour. Therefore, it is reasonable to trace the boundary of the ear for fitting the AR model.

The outer boundary of the ear image is traced by determining the row and column coordinates of the pixel that marks the first change in the grey level in the ear image. This point is to be used as the starting location for the boundary tracing routine provided in MATLB. The boundary of the ear is thus traced as given in FIGURE 2E. The coordinates of this boundary are stored for further processing to obtain AR coefficients.

\subsubsection{Determination of AR Coefficients}

The outer boundary points obtained by boundary tracer gives the sequence of $\mathrm{N}$ points $\left(x_{i}, y_{i}, i=1,2,3 \ldots \ldots . . \mathrm{N}-1\right)$. The sequence that gives contour co-ordinates forms the time series to which AR modeling can be applied to obtain AR coefficients of ear shape. These AR coefficients serve as feature vector which are tested for determining use of AR coefficients for identification of a person.

\section{Classification}

AR coefficients thus obtained can be used for formulation of feature vector. In the present formulation, corresponding to three postures of a person, AR coefficients of ear contour are obtained at rotations: $0^{0}, 45^{\circ}, 90^{\circ}, 135^{\circ}$ and $180^{\circ}$ with respect to reference that is taken as $0^{\circ}$. Ear contours at rotations $0^{\circ}, 45^{\circ}, 90^{\circ}, 135^{\circ}$ and $180^{\circ}$ are shown in Figure 2 (E,G, H,I).This amounts to computation of 15 features in terms of AR coefficients corresponding to three ear images of a person. In order to check the invariance of AR coefficients at three different postures and at five different rotations, the feature vector consists of these 15 AR coefficients are computed at a particular order. In this paper, we compute feature vector at orders ranging from 10 to 100 with an interval of 10 for each subject. To determine if feature vector consisting of AR coefficients can be used as an invariant for determining identity of a person in different postures and rotations that do not change with the change in positions of a person; following co-relation test is performed:

\subsection{Co-relation Test}

In this test, corresponding to 15 features of feature vector of each person correlation within (Intra-correlation) and with respect to feature vector of other persons (Intercorrelations) have been computed. High intra-correlation around the value 1 and very low 
inter-correlation close to 0 has been observed. Typical 3-D scatter plot for some cases is shown in Figure 3. Similarly intra-correlation and inter-correlation among feature vectors at different orders also gives similar results. The results depicted in 3-D scatter plot leads to conclude that AR coefficients at different poses and positions are consistent within it and differ appreciably with feature vectors of other persons. As a result, it can be predicted that these AR coefficients at different orders are useful for the purpose of personal identification.

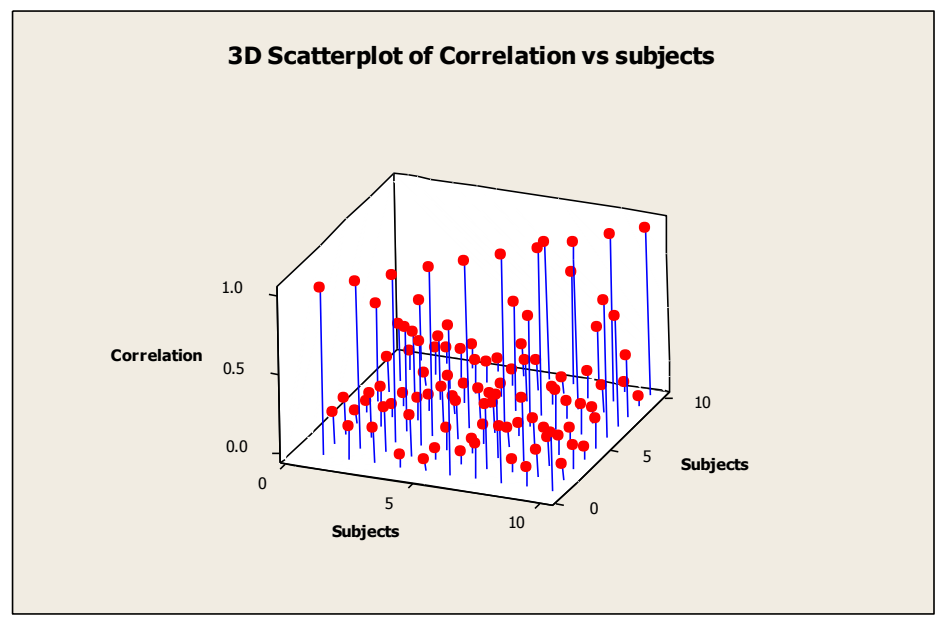

Figure 3. Typical 3D Scatter Plot of Intra and Intercorrelation

\subsection{Recognition Rate}

To validate the findings from correlation test and find the Recognition Rate (RR); feature vector space consisting of 15 features in terms of AR coefficients is broken into two sample sets, training set consisting of 8 features and testing set consisting of 7 features. Training and testing features corresponding to each set are selected at random. Decision is based on computation of Euclidean Distance (ED) between training and testing samples. Thus given a sample from testing set $f_{i}(i=1$ or 2 or $3 \ldots$ or 7$)$ it is compared with training feature vectors $t_{j}(j=1,2, \ldots .8)$ of all the persons in data base on the basis of ED. If ED of $f_{i}$ is least with $t_{j}$ for all values of $j$ when compared with all persons in the data base, it is taken as match. Based on this procedure, matches of testing samples of all subjects have been computed. The Recognition Rate (RR) that gives measure of accuracy is given as:

$$
\mathrm{RR}=\mathrm{k} / \mathrm{n}
$$

Where $\mathrm{k}$ is number of matches and $\mathrm{n}$ is number of test samples tested.

RR at different orders 10, 20, 30,40,50,60,70,80,90,100 is given in Table 1.

Table 1. RR at Different Orders

\begin{tabular}{|l|l|l|l|l|l|l|l|l|l|l|}
\hline \multirow{2}{*}{$\begin{array}{l}\text { RR } \\
\text { in \% }\end{array}$} & \multicolumn{10}{|c|}{ Order } \\
\cline { 2 - 11 } & 10 & 20 & 30 & 40 & 50 & 60 & 70 & 80 & 90 & 100 \\
\cline { 2 - 10 } & 92.8 & 90.8 & 92.77 & 96.77 & 97.22 & 99.11 & 97.11 & 98.66 & 98.2 & 98.5 \\
\hline
\end{tabular}

From the Table I. it is observed that RR is maximum corresponding to order 60 for all subjects. 


\subsection{Performance}

To check the performance of the methodology further, False Acceptance Rate (FAR) and False Rejection Rate (FRR) has also been computed.

\subsubsection{FAR}

Accordingly if a sample matches with some other person on the basis of specified criterion, he/she is considered to have been accepted falsely. Given n samples if number of falsely accepted samples are $\mathrm{f}$.

$$
\mathrm{FAR}=\mathrm{f} / \mathrm{n}
$$

\subsubsection{FRR}

Similarly False Rejection Rate $=\mathrm{r} / \mathrm{n}$

Where $r$ is number of samples rejected falsely. Table 2 shows percentage values of FAR and FRR at different orders.

Table 2. Percentage Values of FAR and FRR at Different Orders

\begin{tabular}{|l|l|l|l|l|l|l|l|l|l|l|}
\hline Order & 10 & 20 & 30 & 40 & 50 & 60 & 70 & 80 & 90 & 100 \\
\hline FAR & 3.2 & 2.8 & 2.5 & 2.3 & 2.4 & 2.1 & 2.9 & 2.7 & 2.5 & 2.1 \\
\hline FRR & 2.2 & 2.2 & 1.7 & 1.6 & 1.5 & 1.6 & 1.4 & 2.0 & 2.2 & 1.9 \\
\hline
\end{tabular}

\section{Comparison with Different Techniques of Ear Biometrics}

The values of RR (Accuracy) of other techniques that use rotation for checking the accuracy of methods used is given in Table 3. From the table following observations are made:

It is observed that in these methods except for $0^{0}$ the accuracy drops with change in angle. The maximum rotation given is $45^{\circ}$ and when the rotation is increased from $5^{\circ}-45^{\circ}$, the accuracy drops gradually to minimum of $42.41 \%$. Exception is $100 \%$ accuracy as given in Wang et al. corresponding to a rotation of $5^{0}$.

In the present method though maximum accuracy is only $99 \%$, however, despite the maximum rotation of $180^{\circ}$ the accuracy does not drop below $90.8 \%$. Thus it is concluded that the percentage accuracy is appreciably high irrespective of change in posture and position of a person. It may be pointed out that other methods use different data base. Therefore, strictly speaking one to one comparison of achieved data base cannot be made. Nevertheless, the proposed method seems to have great potential as performance achieved on standard database is significantly high at all orders of the model.

Table 3. Comparison with Different Techniques of Ear Biometrics

\begin{tabular}{|l|l|l|}
\hline Techniques & Rotation & Accuracy \\
\hline General Fourier Descriptor. & $0^{0}$, & $96 \%$ \\
Abate et al.[25] & $15^{0}$ & $88 \%$ \\
& $30^{0}$ & $88 \%$ \\
\hline Force field Transform \& & $25^{0}$ & $75.3 \%$ \\
NKFDA & $30^{0}$ & $72.2 \%$ \\
Dong \& Mu[23] & $45^{0}$ & $48.1 \%$ \\
\hline IDLLE & $-10^{0}$ to $20^{0}$ & $>80 \%$ \\
Xie \& Mu [37] & $0^{0}$ to $10^{0}$ & $>90 \%$ \\
\hline Local binary pattern \& & $5^{0}$ & $100 \%$ \\
wavelet transform & $20^{0}$ & $92.41 \%$ \\
Wnag et al[32] & $35^{0}$ & $62.66 \%$ \\
& $45^{0}$ & $42.41 \%$ \\
\hline
\end{tabular}




\section{Discussion}

Identification involves computation of stable invariant corresponding to ear shape of a person. In offline applications, it may be sufficient to determine a shape descriptor that is invariant to posture. Accordingly it may be sufficient to check the invariance without any regard to rotation or illumination. However, for online applications the descriptor apart from being invariant with respect to posture needs to be invariant with respect to orientation and illumination as well. In this paper, we have aimed to determine usefulness and efficacy of AR model parameters for on line applications. It is worth mentioning here that if the model parameters are applicable for online applications; they can certainly be applicable for offline applications as well.

Comparison of the method with other techniques which have been checked for variance with respect rotation as well, the proposed method based on AR model reveals superiority in terms of RR, at different possible angles of rotation. The test samples that were used to check the effectiveness of the model were taken at different postures. Therefore model is invariant to postures as well. It is worth pointing out that the model has been fitted to outer boundary of the ear and the boundary does not change with the change in illumination. Therefore, AR model is invariant to illumination as well. In addition, the methodology gives encouraging results in terms of FAR and FRR.

\section{Conclusion}

In this paper AR model has been used for identification of a person on the basis of ear biometrics. To facilitate application of AR model, contour of the ear is obtained using edge detection and binarization. Binarization is followed by contour tracing. The points of the closed contour thus obtained form the time series to which time series model viz; AR model is fitted. The AR coefficients thus obtained form the feature vector.

IIT Delhi ear database has been used to test the efficacy of the model.

The feature vector has been tested at rotation ranging from $0^{\circ}$ to $180^{\circ}$ and at different postures on the basis of Euclidean distance between training and testing set. Maximum RR of $99.11 \%$ at order 60 has been obtained. Thus the results have demonstrated potential of time series modelling for identification of a person

\section{References}

[1] K. W. Bower, H. Worth and P. J. Flynn, "Image understanding for iris biometrics: A survey", Computer vision and image understanding, vol. 1105, (2008) pp. 12.

[2] V. Sravya, P. K. Murthy, R. B. Kallam and B. Srujana, "A Surey on Fingerprint Biometric System”, International Journal of Advanced Research in Computer Science and Software Engineering, vol. 2, no. 4, (2012) April.

[3] S. Goel, A. Kaushik and K. Geol, "A Review paper on Biometrics: Facial Recognition”, International Journal of Scientific Research Engineering \& Technology (IJSRET), vol. 1, no. 017, (2012) August.

[4] Duta, "A survey of Biometric technology based on hand shape", Pattern Recognition, Elsevier, (2009).

[5] Z. Zhang, M. Hu and Y. Wang, "A survey of advances in biometric gait recognition", CCBR'11 Proc. of the $6^{\text {th }}$ Chinese Conference on Biometric recognition, Springer- Verlag Berlin, Heidelberg, (2011), pp. $150-158$.

[6] A. Iannarelli, "Ear identification", Forensic Identification series, Paramount Publishing Company, (1989).

[7] S. Forza, C. Grandi, G. Binelli, M. Tommasi, D. G. Rosati, R. Ferrario and V. F., "Age and Sex-related changes in the normal human ear", Forensic sci. Int), vol. 187, (2009), pp. 1-3.

[8] M. Man, L. Van Der Lugt, C. Maat and G. J. R., "Cross-sectional anthropometric study of the external ear", vol. 52, no. 2, (2007).

[9] M. Burge and W. Burger, "Ear Biometrics", Biometrics: Personal identification in a networked Society, in A.K. Jain, R. Bolle, S. Pankanti, (Eds.), (1998), pp. 273-286.

[10] J. Kaspzzak, "Polish Method of Ear Print Identification", The Information Bulletin for shoeprint/Tool mark Examiners, vol. 9, no. 3, (2003), pp. 20-22.

[11] C. Van Der Lugt, "Ear prints Identification", Elsevier bedrijfsinformatie, Gravenhage, (2001).

[12] C. Van Der Lugt, "Ear Prints in Encyclopaedia of Forensic Science", Academic Press, London, (2000). 
[13] G. Pasescu and E. Tanislav, "Person Identification on the Basis of Ear prints in the Activity of Bucharest Police Department", Information Bulletin for SP/Tm Examiners, vol. 3, no. 3, (1997).

[14] K. H. Pun and Y. S. Moon, "Recent advances in ear biometrics", Proceedings of the $6^{\text {th }}$ IEEE International Conference on Automatic Face and Gesture Recognition (FGR'04), (2004), pp. 7695-2122.

[15] A. Abaza, A. Ross, C. Harrison and M. S. Nixon, "A survey on ear biometrics", ACM Transactions on Embedded Computing System), vol. 9, no. 4, article 39, (2010).

[16] D. J. Hurely, M. S. Nixon and J. N. Carter, "Force field energy functional for image extraction", Image Vis. Comput., vol. 20, no. 5-6, (2002), pp. 311-317.

[17] A. M. Mottaleb and J. Zhou, "Human ear recognition from face profile images", Proc. Of the $2^{\text {nd }}$ international conference on Biometrics ICB. Hong Kong, China, (2006), pp. 786-792.

[18] J. Dong and Z. Mu, "Multi-Pose Ear Recognition Based on Force Field Transformation", In Secymposium on Intelligent Information Technology (IITA), vol. 3, (2008), pp. 771-775.

[19] A. Kumar and C. Wu, "Automated human identification using ear imaging", Pattern recognition, vol. 45, no. 3, (2012), pp. 956-968.

[20] A. F. Abate, M. Nappi and S. Ricciardi, "Ear recognition by means of a rotation invariant descriptor", $18^{\text {th }}$ Int. Conf. On Pattern Recognition, ICPR, vol. 4, (2006), pp. 437-440.

[21] X. Q. Wang, H. Y. Xia and Z. I. Wang, "The research of ear identification based on improved algorithm of moment variants", $3^{\text {rd }}$ Int. Conf. On information and computing (ICIC), (2010), pp. 58.

[22] M. Choras, "Ear biometrics based on geometrical feature extraction", Electronic Letters 'on computer vision and image analysis ELCVIA, vol. 5, no. 3, (2005), pp. 84-95.

[23] M. Choras and R. Chora, "Geometrical algorithms for ear contour shape representation and feature extraction", In proc. of the $6^{\text {th }}$ International Conference on intelligent systems Design and application ISDA, Jinan, China, (2006).

[24] G. D. Lowe, "Object recognition from local scale invariant features", IEEE Int. Conf. on computer vision (ICCV), vol. 2, (1999), pp. 1150-1157.

[25] J. D. Bustard and M. S. Nixon, "Towards unconstrained ear recognition from two dimensional images", Syst. Man Cybern A syat Hum, vol. 40, (2010), pp. 486.

[26] Y. Guo and Z. Xu, "Ear Recognition Using a New Local Matching Approach", $15^{\text {th }}$ IEEE International Conference on Image Processing (ICIP), (2008), pp. 289-292.

[27] Z. Q. Wang and X. D. Yan, "Multi-Scale Feature Extraction Algorithm of Ear Image", International Conference on Electric Information and Control Engineering (ICEICE), (2011), pp. 528.

[28] H. Liu and J. Yan, "Multi-view Ear Shape Feature Extraction and Reconstruction", Third International IEEE Conference on Signal-Image Technologies and Internet- Based System (SITIS), (2007), pp. 652 658.

[29] L. Yuan and Z. Mu, "Ear Recognition Based on 2D Images", First IEEE International Conference on Biometrics: Theory, Applications, and Systems (BTAS), (2007), pp. 15.

[30] L. Lu, Z. Xiaoxun, Z. Youdong and J. Yunde, "Ear Recognition Based on Statistical Shape Model", First International Conference on Innovative Computing, Information and Control (ICICIC), (2006), pp. 353-356.

[31] H. Zhang and Z. Mu, "Compound Structure Classier System for Ear Recognition", IEEE International Conference on Automation and Logistics (ICAL), (2008), pp. 2306-2309.

[32] Z. Xie and Z. Mu, "Ear Recognition Using LLE and IDLLE Algorithm", $19^{\text {th }}$ International Conference on Pattern Recognition (ICPR), (2008), pp. 1-4.

[33] I. Naseem, R. Togneri and M. Bennamoun, "Sparse Representation for Ear Biometrics", Bebis G, Boyle R, Parvin B, Koracin D, Remagnino P, Porikli F, et al., "editors, Advances in Visual Computing of Lecture Notes in Computer Science, Springer Berlin / Heidelberg, vol. 5359, (2008), pp. 336-345.

[34] A. Pflug and C. Busch, "Ear biometrics: A survey of detection, feature extraction and recognition methods", IET biometrics, vol. 1, issue 2, (2012), pp. 114-129.

[35] K. Masaoud, S. Algabary, K. Omar, J. Nordin, S. Noorul and H. S. Abdullah, "A review paper on ear recognition techniques: models, algorithms and methods", Australian Journal of basic and applied sciences, vol. 7, no. 11, (2013), pp. 411-421.

[36] C. K. Chow and T. Kaneko, "Boundary detection of radiographic images by a threshold Method", Front. Pattern Recognition, (1972), pp. 61-82.

[37] R. M. Haralick, "Edge and region analysis for digital image data", Computer Graphics image Processing, (1980), pp. 60-73.

[38] R. Navatia and K. R. Babu, "Linear feature and description", Computer Graphics Image Processing, vol. 13, (1980), pp. 257-269.

[39] T. O. Binford, "Inferring surfaces from images", Artificial Intell., vol. 17, (1981) August, pp. 205-244.

[40] D. C. Marr and E. Hildreth, "Theory of edge detection", Proc. Royal Society of London, vol. 207.

[41] K. S. Shanmugam, F. M. Dicky and J. A. Green, "An optimal frequency domain filter for edge detection digital images”, IEEE Trans. Pattern Anal. Machine Intell., PAMI-1, (1979) January, pp. 37-49.

[42] J. Canny, "A computational approach to edge detection", IEEE Trans. Pattern Anal. Machine Intell, PAMI-8, (1986) November. 
[43] M. Poulos, M. Rangoussi, V. Chrissicopoulos and A. E. Gelou, "Person identification based on parametric processing on the EEG", Proc. Of the $6^{\text {th }}$ international Coonference on Electronics Ciruiys and Systems, vol. 1, (1999), pp. 281-286.

[44] R. B. Paranjape, "Electroencephalogram as a biometric", Proceedings of the Canadian Conference on Electrical and Computer Engineering, vol. 2, (2001), pp. 1363-1366.

[45] R. L. Kashyap and R. Chellapa, "Stochastic models for closed boundary analysis: Representation and reconstruction”, IEEE Trans. Inform. Theory, vol. IT-27, (1981), pp. 627-635.

[46] S. R. Dubois and F. H. Glanz, "An autoregressive model approach to two dimensional shape classification”, IEEE Trans. Pattern Anal. Machine Intell., PAMI-8, (1986) January, pp. 55-66.

[47] A. H. Mir, M. Handmandlu and S. N. Tandon, "Description of shapes in CT images the usefulness of time-series modelling techniques for identifying organs", IEEE Eng. Med, Biol. Mag, vol. 18, (1999), pp. 79-84.

\section{Authors}

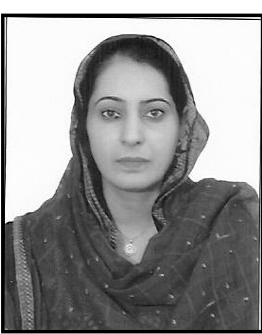

Farida Khursheed, has done her B.E in Electronics \& Communication Engineering (ECE) and M.Tech in Communication and Information Technology in the year 2007 both from National Institute of Technology (NIT), Srinagar, Jammu and Kashmir. India. Presently she is working as Associate Professor in the Department of ECE at NIT Srinagar, India. She has guided a number of M.Tech thesis and B.Tech projects. She has a number of publications in her credit. Currently she is perusing her Ph.D in the area of Biometrics. Her areas of interest are Biometrics, Image processing and Security.

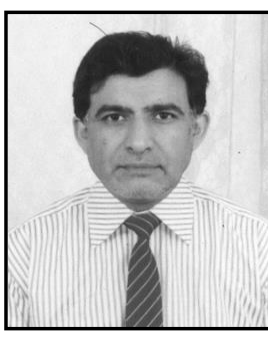

Ajaz Hussain Mir, has done his B.E in Electrical Engineering with specialization in Electronics \& Communication Engineering (ECE) .He did his M.Tech in Computer Technology and Ph.D both from IIT Delhi in the year 1989 and 1996 respectively. He is Chief Investigator of Ministry of Communication and Information Technology, Govt. of India project: Information Security Education and Awareness (ISEA). He has been guiding Ph.D and M.Tech thesis related to the area of Security and other related areas and has a number of International publications to his credit Presently he is working as Professor in the Department of Electronics \& Communication Engineering at NIT Srinagar, India. His areas of interest are Biometrics, Image processing, Security, Wireless Communication and Networks. 